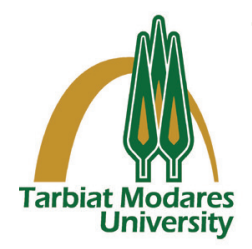

\title{
Management of a Pregnant Woman during the Pandemic of COVID-19: A Review
}

\section{A R T I C L E I N F O}

\section{Article Type}

Review Article

\section{Authors}

Arezoo Nabizadeh, $M S c^{1}$

Mehrdad Ravanshad, $P h D^{1^{*}}$

Elham Ahmadi, $M S c^{1}$

Haniyeh Olad, $B S c^{1}$

Hadiseh Bagheri, $B S c^{1}$

Monireh Hosseini, $B S C^{1}$

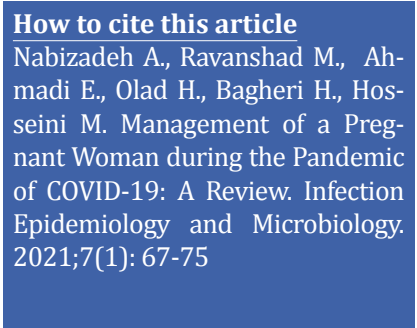

${ }^{1}$ Department of Virology, Faculty of Medical Sciences, Tarbiat Modares University, Tehran, Iran.

\section{* Correspondence}

Address: Department of Virology, Faculty of Medical Sciences, Tarbiat Modares University, Tehran, Iran. ravanshad@modares.ac.ir

\section{A B S T R A C T}

Background: In late December 2019, a new coronavirus called SARS-CoV-2, as the causative agent of COVID-19 (coronavirus disease 2019) pneumonia, began to spread from Wuhan, China. The SARS-CoV-2 virus is currently threatening the world's public health and so far, no specific and effective treatment has been found for the disease. Due to the physiological changes in the body of pregnant women during pregnancy, the occurrence of infection in pregnant women could affect the health of both mother and her fetus. The present paper aimed to study the findings related to the effects of coronavirus on pregnant women and their fetuses during pregnancy, diagnostic methods, care protocols in pregnant women, and postpartum care. Methods: The information included in this review was extracted from articles, published protocols, and valid statistics. In this study, articles indexed in Latin databases (PubMed, Google Scholar, Science Direct, Embase, Scopus), clinical protocols, and reports of reputable health organizations from January to august 2020 were reviewed using a combination of the following keywords: COVID-19, SARS-CoV-2, pregnant woman, and pregnancy.

Conclusion: Due to the global prevalence of this virus, protocols and health care should be applied for pregnant women in accordance with other people. It is also necessary to take preventive measures at the community level. In this regard, the management of pregnant women with COVID-19 infection is of particular importance during the pandemic period of the disease. However, more extensive studies are required to validate these principles. This review summarized the precautions that should be taken before and after delivery.

\section{Keywords: COVID-19, Pregnancy, Pregnant, SARS-CoV-2.}

\section{CITATION LINKS}

[1] Hussein J. COVID-19: What implications for sexual and ... [2] Riley T, Sully E, Ahmed Z, Biddlecom A. Estimates of the potential ... [3] Khurana A, Sharma KA, Bachani S, Sahi G, Bhatt RK, Sahani B, et al. SFM India Oriented ... [4] Nunes MC, Madhi SA. Influenza vaccination during pregnancy ... [5] Dashraath P, Jeslyn WJL, Karen LMX, Min LL, Sarah L, Biswas A, et al. Coronavirus disease... [6] Shi Y, Wang Y, Shao C, Huang J, Gan J, Huang X, et al. COVID-19 infection: the perspectives on... [7] Zhang Y, Wang Y, Li MQ, Duan J, Fan DX, Jin LP. IL-25 promotes... [8] Zhao X, Jiang Y, Zhao Y, Xi H, Liu C, Qu F, et al. Analysis of the... [9] Muldoon KM, Fowler KB, Pesch MH, Schleiss MR. SARS-CoV-2: Is it the newest spark... [10] Zhu H, Wang L, Fang C, Peng S, Zhang L, Chang G, et al. Clinical... [11] Panahi L, Amiri M, Pouy S. Risks of novel coronavirus disease... [12] Qiao J. What are the risks of COVID-19 infection in... [13] Hasnain M, Pasha MF, Ghani I, Budiarto R. Protection... [14] Xi J, Xu K, Jiang P, Lian J, Hao S, Jia H, et al. Virus strain of... [15] Liu D, Li L, Wu X, Zheng D, Wang J, Yang L, et al. Pregnancy and perinatal... [16] Wu Z, McGoogan JM. Characteristics of and important lessons from... [17] Baraldi E, Filippone M. Chronic lung disease after premature... [18] Chen H, Guo J, Wang C, Luo F, Yu X, Zhang W, et al. Clinical characteristics... [19] Rasmussen SA, Smulian JC, Lednicky JA, Wen TS, Jamieson DJ. Coronavirus... [20] Chen D, Yang H, Cao Y, Cheng W, Duan T, Fan C, et al. Expert consensus for... [21] Liu H, Wang L-L, Zhao S-J, Kwak-Kim J, Mor G, Liao A-H. Why are... [22] Dong L, Tian J, He S, Zhu C, Wang J, Liu C, et al. Possible vertical... [23] Procianoy RS, Silveira RC, Manzoni P, Sant'Anna G. Neonatal COVID-19: little evidence... [24] Liu H, Liu F, Li J, Zhang T, Wang D, Lan W. Clinical and CT imaging features... [25] Ahmed M. HRCT Chest Manifestations of... [26] Cascella M, Rajnik M, Cuomo A, Dulebohn SC, Di Napoli R. Features, evaluation and... [27] Lowe B, Bopp B. COVID-19 vaginal delivery-a case report. Australian and... [28] Zeng H, Xu C, Fan J, Tang Y, Deng Q, Zhang W, et al. Antibodies in... [29] Mullins E, Evans D, Viner R, O'Brien P, Morris E. Coronavirus in pregnancy and... [30] Mimouni F, Lakshminrusimha S, Pearlman SA, Raju T, Gallagher PG, Mendlovic J. Perinatal... [31] Elshafeey F, Magdi R, Hindi N, Elshebiny M, Farrag N, Mahdy S, et al. A systematic... [32] Sentilhes L, De Marcillac F, Jouffrieau C, Kuhn P, Thuet V, Hansmann Y, et al. Coronavirus...

\section{Article History}

Received: September 25,2020

Accepted: December 25,2020

Published: January 23,2021

Copyright(C) 2021, TMU Press. This open-access article is published under the terms of the Creative Commons Attribution-NonCommercial 4.0 International License which permits Share (copy and redistribute the material in any medium or format) and Adapt (remix, transform, and build upon the material) under the Attribution-NonCommercial terms. 


\section{Introduction}

In late December 2019, a new coronavirus called SARS-CoV-2, as the causative agent of 2019 new coronavirus (2019$\mathrm{nCoV}$ ) pneumonia, began to spread from Wuhan, China. Some cases of coronavirus infections have already been reported, including infections caused by SARS-CoV (Severe Acute Respiratory Syndrome) in 2002-2003 and MERS-CoV (Middle East Respiratory Syndrome) in 2012.

Coronaviruses have a single-stranded RNA genome that causes the disease, ranging from the common cold to severe and deadly respiratory illness. The average duration of the disease symptoms is 5 days (ranging from 2 to 14 days). The average age of hospitalized patients has been reported to be 46-59 years, of which one-third to one-half have an underlying disease. Frequent manifestations include fever, cough, myalgia, headache, and diarrhea. The virus is mainly transmitted through respiratory droplets or humanto-human transmission.

SARS-CoV-2 is currently threatening the world's public health and so far, no specific and effective treatment has been found for the disease.

The high prevalence and transmission of the virus could affect sexual and reproductive health in a variety of ways ${ }^{[1,2]}$.

Management of pregnant women with COVID-19 infection should be performed by a multidisciplinary team including obstetricians and gynecologists, anesthesiologists, midwives, virologists, microbiologists, neonates, and infectious disease specialists.

\section{Screening}

During the COVID-19 epidemic, a detailed history should be taken of all pregnant women, seeking routine items such as recent travel, employment, and significant group contact (TOCC: Travel, Occupation,
Significant Contact and Cluster of case). Pregnant women with these risk-factors should be placed in an isolated room because they need further evaluation. Pregnant women with known risk factors of TOCC without symptoms of COVID-19 infection or with mild symptoms should delay routine pregnancy care and routine ultrasound evaluation for 14 days ${ }^{[3]}$.

The examination of patients with suspected SARS-CoV-2 infection and the confirmation of this viral infection should be performed in level-3 hospitals with special protective facilities and equipment, and if the disease is confirmed, negative pressure should be transferred to the ICU (intensive care unit).

\section{SARS-CoV-2 virus and pregnancy}

Pregnancy is considered as a risk factor for respiratory diseases, including influenza. On the other hand, due to frequent visits to health centers, the risk of SARS-CoV-2 infection increases in pregnant women due to physiological changes during pregnancy. Therefore, preventive care is of particular importance ${ }^{[1,2,4]}$.

Pregnant women are at high risk of physiological changes and suppression of the immune system. Anatomically, changes occurring in the respiratory system and respiratory organs of pregnant women increase their need to inhale more air. On the other hand, airborne particles could be easily contaminated by SARS-CoV-2 in respiratory droplets. In addition, changes in the nasal mucosa due to progesterone secretion during pregnancy may cause the virus to stick to the upper respiratory tract, making it difficult to clear. Increased ACE2 (angiotensin-converting enzyme 2) in pregnancy may play a role in increasing susceptibility to SARS-CoV-2. Physiological changes in pregnant women not only increase their susceptibility to the virus but also increase the severity of the disease. 
In terms of changes in the immune system during pregnancy, the Th2/Th1 balance is diverted toward Th2, which is the cause of change in environmental responses to respiratory viral infection and disease. Increased estrogen and progesterone in the first trimester of pregnancy leads to a decrease in CD4 + and CD8 + T cells.

Previous studies conducted on SARS and MERS infections during pregnancy have shown complications such as intrauterine growth restriction, preterm delivery, miscarriage, and maternal death [5-10]. Of course, the effects of SARS-CoV-2 infection during pregnancy are not yet fully understood, but high fever in the first trimester could increase the risk of certain birth defects. There are also few reports of fetal distress and preterm delivery ${ }^{[11]}$.

\section{Protection of pregnant women during COVD-19 epidemic}

During pregnancy, the body's immune system naturally weakens to allow the pregnancy to continue successfully. This means that pregnant women may get sick faster. It is recommended that all pregnant women get the flu shot. The vaccine protects the mother and baby from the flu, but it does not protect them against SARS-CoV-2. It is also highly recommended that pregnant women, like normal people, do the following instructions to prevent COVID-19:

- Washing hands regularly with soap and water.

- Observing the social distance between themselves and other people and avoiding crowded spaces.

- Avoiding contact of contaminated hands with eyes, nose, and mouth.

- Observing respiratory hygiene.

- Talking to their healthcare provider if they notice symptoms such as fever and cough [12, 13].

\section{Childbirth care}

During the delivery process, the number of doctors and staff should be kept to a minimum, and all staff should be protected from the virus and use N95 masks, helmets, safety goggles, and protective clothing. Childbirth should be performed in a negative pressure respiratory isolation environment (a negatively charged arch has less pressure than the adjacent section, which prevents air flow to the adjacent rooms and sections).

Cesarean section should be performed according to the relevant delivery indications. Proper planning should be done immediately for patient transfer in order to minimize contact with other patients in the hospital ${ }^{[14,15]}$.

\section{Child care after birth to prevent disease}

Due to frequent contact with health centers and increased risk of SARS-CoV-2 infection, preventive care is extremely important; in some cases, using a mask is recommended for the child ${ }^{[16,17]}$.

\section{Mother during pregnancy with Covid19}

It is recommended that pregnant women with suspected respiratory illness be transferred to a single room immediately after being visited by a doctor, medical examinations be minimized, and patients be kept at home as much as possible. In special cases, doctors recommend preterm delivery. In such cases, corticosteroids are recommended by doctors to accelerate fetal lung maturation. Examination of fetal heart rate using ultrasound or electrical procedures should be monitored regularly according to fetal age. Contamination of immune fluid with COVID-19 infection in an infected mother is usually not examined unless in special circumstances [18, 19]. COVID-19 infection is associated with cytokine storms, which are associated with increased IL2, IL7, IL10, GCSF, GIFN, inflammatory MQ1, and $\alpha$ TNF levels. 
SARS-CoV-2-induced elevated cytokines were shown to probably cause more severe inflammation in pregnant women in the first and third trimesters. Maternal inflammation as a resultof aviralinfection during pregnancy could affect the development of fetal brain. An abnormal increase in peripheral blood level of $\alpha$ TNF could affect the fetus> early growth. Overall, these findings suggest that even if the virus does not reach the fetus, the fetus would be affected by the motherss immune response to the infection ${ }^{[20,21]}$.

\section{Vertical transmission of COVID-19 virus from pregnant mother to infant}

In cases where COVID-19 disease is confirmed in a pregnant woman, fetal anatomy examination is performed monthly. Some Chinese women with symptoms of the disease gave birth prematurely. It was not known whether the coronavirus caused premature birth or mothers had to give birth prematurely due to their poor condition. Also, after the birth, a COVID-19 diagnostic test was performed for infants to examine vertical transmission of infection to the infant. Because ACE2 receptor is widely expressed in placenta, there is a possibility of vertical transmission. However, no evidence of vertical transmission of SARSCoV-2 has been reported so far, the new coronavirus is highly contagious and could not be transmitted internally ${ }^{[5]}$.

In a digital study of a baby born to a mother with COVID-19, 2 hours after birth, the baby's antibodies and cytokines levels were abnormal. Given that IgM antibodies were not transmitted to the fetus through the placenta, the IgM levels indicated that the baby was infected in the womb ${ }^{[22,23] .}$

\section{Imaging during pregnancy}

Pregnant women suspected of having COVID-19 infection should have a CT scan of the chest, as a primary diagnostic tool for COVID-19 infection in epidemic areas. Imaging of pregnant mothers should be done at specific time intervals and separately from other patients, and in cases with suspected COVID-19 disease, the site should be cleaned with disinfectant after imaging ${ }^{[15,24]}$.

In case of emergency, due to the importance of early diagnosis and treatment, low-dose HRCT should be performed according to the relevant physician. In this regard, the use of lead protector is necessary to protect the mother and fetus ${ }^{[25]}$.

\section{Treatment}

Approved treatments for COVID-19 cases with mild symptoms:

- Maintaining fluid and electrolyte balance and using symptomatic therapies such as nonsteroidal anti-inflammatory drugs are recommended to reduce fever and pain.

- Careful monitoring of vital signs such as oxygen saturation, arterial blood gases, chest imaging, blood cell counts, kidney and liver function, and coagulation factors are recommended.

- Evaluation of fetal heart rate with the help of cardiography and examination of fetal growth and amniotic fluid volume with the help of ultrasound, and if necessary with umbilical artery Doppler, are recommended. - Bacterial infection should be monitored, and antibiotics should be used in a timely manner if there is a secondary bacterial infection. It is worth noting that the disease monitoring devices should be carefully disinfected before use.

\section{Treatment of confirmed cases of COVID-19 with severe symptoms:} - Hydration therapy, oxygen therapy, and chest physiotherapy should be performed in an isolated room with negative pressure.

- If there is a secondary bacterial infection, the use of appropriate antibiotics in combination with antiviral therapy is 
recommended. The primary line of antibiotic treatment for bacterial pneumonia during COVID-19 is amoxicillin and in severe cases, ceftriaxone is recommended.

- Monitoring of blood pressure and fluid balance in patients with symptoms of septic shock as well as resuscitation of fluid, inotropic, and norepinephrine are necessary to maintain arterial blood pressure of about $60 \mathrm{~mm} \mathrm{Hg}$ and lactate levels of less than 2 $\mathrm{mM} / \mathrm{L}^{[26]}$.

- Most pregnant women need about 95 percent or more oxygen to keep their baby hydrated. Also, to maintain oxygen saturation of about $95 \%$ or more, auxiliary oxygen should be used, and ventilation should be performed under the supervision of an anesthesiologist and obstetrician.

- Evaluation of fetal heart rate with the help of cardiography and examination of fetal growth and amniotic fluid volume with the help of ultrasound, and if necessary with umbilical artery Doppler, are recommended $[15,20]$

\section{Antiviral therapies}

Many antiviral drugs are contraindicated during pregnancy because they are teratogenic. For example, ribavirin is contraindicated during pregnancy because it could cause abnormalities in the fetus. Hydroxychloroquine is a new anticongestion drug that is structurally different from chloroquine. The side effects of hydroxychloroquine are significantly less than those of chloroquine. The WHO has also not reported any side effects of chloroquine on pregnancy, childbirth, or infants. According to research, hydroxychloroquine is assumed to have the potential to be used as an effective drug for pregnant women with COVID-19.

IFN- $\alpha$ also does not increase the risk of miscarriage, childbirth, or premature birth. Therefore, in the case of COVID-19 during
pregnancy,IFNispredictedtobeeffectiveandsafe. Lopinavir / Ritonavir, which was used to fight HIV, is therefore safer than other antiviral drugs. Lopinavir / Ritonavir is the preferred antiviral therapy for pregnant women with COVID-19. Host-directed therapy: HDT has been shown to be safe and effective in combination with commonly used drugs such as metformin, glitazone, and atorvastatin ${ }^{[8]}$.

\section{Giving birth}

COVID-19 infection by itself is not a sign of childbirth, unless maternal oxygen delivery needs to be improved. The timing and manner of delivery should be decided individually according to the patient's clinical condition, gestational age, and fetal condition.

The hospital should be equipped with a dedicated operating room with negative pressure and a neonatal isolation ward, and all medical personnel who provide the necessary care for the patient infected with COVID19 should be fitted out with personal protective equipment.

Initially, the Centers for Disease Control and Prevention recommends that pregnant women suspected of having COVID-19 be tested for COVID-19 before entering the obstetrics and gynecology department in order to take appropriate steps to control the infection. Care during childbirth is similar to the maternity protocol for mothers who are negative for COVID-19. All surgical devices must have the COVID-19 label to be disinfected separately after cesarean section [19].

Pregnant women who recover quickly do not need preterm labor. In the last trimester, it is best for the fetus to grow fully. According to some studies, COVID-19 infection during pregnancy could cause complications for both mother and fetus, such as preterm labor, respiratory problems, and fetal distress. Other pregnant women with COVID-19 showed 
other complications, such as preeclampsia, premature rupture of the bladder, and irregular contractions, indicating early labor intervention. Whether or not these side effects are related to COVID-19 needs further investigations

If the mother has stable conditions, vaginal delivery is recommended; but in case of complications such as shock or loss of vital organs, cesarean section should be performed immediately.

No studies have been done on vaginal delivery yet, but it is still better than long procedures such as cesarean section.

If one has a cesarean section, epidural anesthesia and local anesthesia are not prohibited. In addition, it is recommended that patients with general anesthesia wear a mask during childbirth, and that the closure of the baby's umbilical cord should not be delayed to minimize the infection.

Also, the necessary tests of the placenta sample should be performed in accordance with the hygienic regulations, and at the end, the placenta sample of the infected mother should be disposed in biological waste in accordance with hygienic principles and regulations.

Maternity ward staff should also be evaluated periodically for SARS-CoV-2 infection ${ }^{[15,19]}$.

\section{Postpartum care}

Complete separation of the baby from the mother is not recommended, unless the strict precautions for the isolation of mother are discontinued.

Breastfeeding for mothers with or suspected of having COVLD-19: No virus has been detected in breast milk of mothers with SARS infection. Given the presence of antibodies in breast milk, it is recommended to minimize the mother's contact with the baby for 14 days.

It is recommended that the mother wash her hands before touching the baby and use a mask when feeding the baby either by breastmilk or bottle. Although there is a possibility of transmitting antibodies from breast milk to infants, limited evidence has been reported in this regard so far [18].

Limited studies have been performed on women with COVID-19 and other coronavirus infections, including SARS; however, no virus has been detected in breast milk so far, and it is not well known if mothers with COVID-19 could transmit it to their babies through breastfeeding.

It is recommended that if the patient is asymptomatic or has mild symptoms, breastfeeding and room-mating of mother and her baby be done in coordination with health care providers.

Because the main concern is that the virus may be transmitted through respiratory droplets instead of breast milk, breastfeeding mothers must wash their hands and wear a three-layer surgical mask before contact with the baby. In the case of a mother and baby roommate, the baby's bed should be at least 2 meters away from the mother, and a physical barrier such as a curtain should be used.

If the mother is very ill, separation seems to be the best option, trying to get the breast milk out in order to maintain milk production. Precautions should be taken when cleaning breast pumps.

Breast milk protects the baby against many diseases. But in rare cases, breastfeeding is not recommended. The US Centers for Disease Control and Prevention (CDC) has not provided any specific guidelines for breastfeeding of mother infected with similar viruses, such as SARS or MERS. The US Centers for Disease Control and Prevention (CDC) recommends that a mother with influenza continue to breastfeed outside the postpartum position by following health tips to prevent the transmission of the disease to the baby ${ }^{[27,28]}$. 
If the child is infected through contact with an infected mother

There is still no comprehensive information on the transmission of coronavirus from infected mothers to infants or how the disease affects the infant.

On the other hand, limited studies have been performed on infants with COVID-19, indicating that the symptoms of the disease are very mild in infants. The youngest infant infected was reported to be a 36-hour-old baby ${ }^{[28]}$.

Studies have shown that babies whose test results are positive and show symptoms from 5 to 17 days are often infected through the infected mother's cough during breastfeeding, embracement of the baby by relatives, or through the contaminated environment.

The US CDC recommends that the baby not need to be separated from the mother, but if the mother is definitely infected with COVID-19 infection, the baby should be placed in a separate room or, if not possible, at a distance of 2 meters ${ }^{[29]}$.

According to algorithm Number 1, If the result of the mother's test is positive, the use of a mask is necessary for the mother, and in order to reduce the risk of exposure, the number of staff and nurses in the delivery room should be minimized, and the baby should be separated from the mother immediately after delivery and kept in a separate room. The recommended test for a baby's mouth and throat while swallowing is performed 24 hours after birth, and if the test is negative, it is repeated 48 hours after birth. If the test is negative, the child could be discharged, and if the test is positive, and the baby is asymptomatic, the baby may be discharged but may be quarantined at home. These guidelines vary with the evidence and symptoms of the disease.

\section{Recommended management for pregnant women with suspected COVID-19 disease}

If a mother's tests are positive, the mother should use a mask. To reduce the risk of exposure, the number of crew and nurses in the delivery room should be kept to a minimum. The baby is separated from the mother immediately after delivery and placed in a separate room for testing. The recommended test is nasopharyngeal and throat swabs testing performed 24 hours after birth and repeated 48 hours after birth. If the tests are negative, and the child is asymptomatic, he or she could be discharged. If the tests are positive, but the infant is asymptomatic, the child may be discharged but may be quarantined at home. If possible, people over 60 and those with comorbidities should not take care of these babies. If marked, the child needs NICU. These instructions could be changed with evidence [30].

\section{Conclusion}

The COVID-19 epidemic still affects many countries and people around the world and could affect the health of most people.

Although pregnancy is not very affected by the COVID-19 disease, failure to take necessary and timely precautions could lead to side effects on mother and fetus. Therefore, managing pregnancy period during the pandemic is of particular importance.

Accordingly, the protocols and care applied to other groups should be applied to pregnant women; on the other hand, taking preventive measures is important for them, and this group of people should be controlled by monitoring systems [15, 31, 32].

Acknowledgments: This work was supported by Deputy of Research and Technology, Tarbiat Modares University, Tehran, Iran.

Ethical Permission: Not applicable.

Authors Contribution: Conceptualization: AN and MR; Data curation and formal analysis: All Authors; Investigation: AN and MR; 
Methodology and project administration: MR; Supervision: MR; Validation: AN and MR; Writing of original draft: AN and MR; Writing, reviewing, and editing: All Authors. Conflict of interest: None.

Funding: None.

Consent to participate:None.

\section{References}

1. Hussein J. COVID-19: What implications for sexual and reproductive health and rights globally? : Taylor \& Francis; 2020.

2. Riley T, Sully E, Ahmed Z, Biddlecom A. Estimates of the potential impact of the COVID-19 pandemic on sexual and reproductive health in low-and middle-income countries. International Perspectives on Sexual and Reproductive Health. 2020;46:73-6.

3. Khurana A, Sharma KA, Bachani S, Sahi G, Bhatt RK, Sahani B, et al. SFM India Oriented Guidelines for Ultrasound Establishments During the COVID 19 Pandemic. Journal of Fetal Medicine. 2020:1-7.

4. Nunes MC, MadhiSA. Influenzavaccination during pregnancy for prevention of influenza confirmed illness in the infants: a systematic review and meta-analysis. Human vaccines \& immunotherapeutics. 2018;14(3):758-66.

5. Dashraath P, Jeslyn WJL, Karen LMX, Min LL, Sarah L, Biswas A, et al. Coronavirus disease 2019 (COVID-19) pandemic and pregnancy. American journal of obstetrics and gynecology. 2020.

6. Shi Y, Wang Y, Shao C, Huang J, Gan J, Huang $X$, et al. COVID-19 infection: the perspectives on immune responses. Nature Publishing Group; 2020.

7. Zhang Y, Wang Y, Li MQ Duan J, Fan DX, Jin LP. IL-25 promotes Th2 bias by upregulating IL-4 and IL-10 expression of decidual $\gamma \delta \mathrm{T}$ cells in early pregnancy. Experimental and therapeutic medicine.
2018;15(2):1855-62.

8. Zhao X, Jiang Y, Zhao Y, Xi H, Liu C, Qu F, et al. Analysis of the susceptibility to COVID-19 in pregnancy and recommendations on potential drug screening. European Journal of Clinical Microbiology \& Infectious Diseases. 2020:1.

9. Muldoon KM, Fowler KB, Pesch $\mathrm{MH}$, Schleiss MR. SARS-CoV-2: Is it the newest spark in the TORCH? Journal of Clinical Virology. 2020:104372.

10. Zhu H, Wang L, Fang C, Peng S, Zhang L, Chang G, et al. Clinical analysis of 10 neonates born to mothers with 2019-nCoV pneumonia. Translational pediatrics. 2020;9(1):51.

11. Panahi L, Amiri M, Pouy S. Risks of novel coronavirus disease (COVID-19) in pregnancy; a narrative review. Archives of Academic Emergency Medicine. 2020;8(1).

12. Qiao J. What are the risks of COVID-19 infection in pregnant women? The Lancet. 2020;395(10226):760-2.

13. Hasnain M, Pasha MF, Ghani I, Budiarto R. Protection challenges of pregnant women against vertical transmission during COVID-19 epidemic: a narrative review. American journal of infection control. 2020.

14. Xi J, Xu K, Jiang P, Lian J, Hao S, Jia H, et al. Virus strain of a mild COVID-19 patient in Hangzhou representing a new trend in SARS-CoV-2 evolution related to Furin cleavage site. medRxiv. 2020.

15. Liu D, Li L, Wu X, Zheng D, Wang J, Yang L, et al. Pregnancy and perinatal outcomes of women with coronavirus disease (COVID-19) pneumonia: a preliminary analysis. American journal of roentgenology. 2020:1-6.

16. Wu Z, McGoogan JM. Characteristics of and important lessons from the coronavirus disease 2019 (COVID-19) outbreak in China: summary of a report 
of 72314 cases from the Chinese Center for Disease Control and Prevention. Jama. 2020;323(13):1239-42.

17. Baraldi E, Filippone M. Chronic lung disease after premature birth. New England Journal of Medicine. $2007 ; 357$ (19):1946-55.

18. Chen H, Guo J, Wang C, Luo F, Yu X, Zhang $\mathrm{W}$, et al. Clinical characteristics and intrauterine vertical transmission potential of COVID-19 infection in nine pregnant women: a retrospective review of medical records. The Lancet. 2020;395(10226):809-15.

19. Rasmussen SA, Smulian JC, Lednicky JA, Wen TS, Jamieson DJ. Coronavirus Disease 2019 (COVID-19) and Pregnancy: What obstetricians need to know. American journal of obstetrics and gynecology. 2020.

20. Chen D, Yang H, Cao Y, ChengW, Duan T, Fan $\mathrm{C}$, et al. Expert consensus for managing pregnant women and neonates born to mothers with suspected or confirmed novel coronavirus (COVID-19) infection. International Journal of Gynecology \& Obstetrics. 2020;149(2):130-6.

21. Liu H, Wang L-L, Zhao S-J, Kwak-Kim J, Mor G, Liao A-H. Why are pregnant women susceptible to viral infection: an immunological viewpoint? Journal of reproductive immunology. 2020:103122.

22. Dong L, Tian J, He S, Zhu C, Wang J, Liu $\mathrm{C}$, et al. Possible vertical transmission of SARS-CoV-2 from an infected mother to her newborn. Jama. 2020.

23. Procianoy RS, Silveira RC, Manzoni P, Sant'Anna G. Neonatal COVID-19: little evidence and the need for more information. Jornal de Pediatria. 2020.

24. Liu H, Liu F, Li J, Zhang T, Wang D, Lan W. Clinical and CT imaging features of the COVID-19 pneumonia: Focus on pregnant women and children. Journal of infection. 2020.

25. Ahmed M. HRCT Chest Manifestations of COVID-19 Pneumonia in a Time Series.

26. Cascella M, Rajnik M, Cuomo A, Dulebohn SC, Di Napoli R. Features, evaluation and treatment coronavirus (COVID-19). Statpearls [internet]: StatPearls Publishing; 2020.

27. Lowe B, Bopp B. COVID-19 vaginal delivery-a case report. Australian and New Zealand Journal of Obstetrics and Gynaecology. 2020.

28. Zeng H, Xu C, Fan J, Tang Y, Deng Q Zhang $\mathrm{W}$, et al. Antibodies in infants born to mothers with COVID-19 pneumonia. Jama. 2020.

29. Mullins E, Evans D, Viner R, O'Brien P, Morris E. Coronavirus in pregnancy and delivery: rapid review. Ultrasound in Obstetrics \& Gynecology. 2020;55(5):586-92.

30. Mimouni F, Lakshminrusimha S, Pearlman SA, Raju T, Gallagher PG, Mendlovic J. Perinatal aspects on the covid-19 pandemic: a practical resource for perinatal-neonatal specialists. Journal of Perinatology. 2020:1-7.

31. Elshafeey F, Magdi R, Hindi N, Elshebiny M, Farrag N, Mahdy S, et al. A systematic scoping review of COVID-19 during pregnancy and childbirth. International Journal of Gynecology \& Obstetrics. 2020;150(1):47-52.

32. Sentilhes L, De Marcillac F, Jouffrieau C, Kuhn P, Thuet V, Hansmann Y, et al. Coronavirus disease 2019 in pregnancy was associated with maternal morbidity and preterm birth. American journal of obstetrics and gynecology. 2020. 